\title{
CLINICO-MICROBIOLOGY OF CHOLESTEATOMA OBTAINED DURING MASTOID SURGERY IN PATIENTS WITH CHRONIC SUPPURATIVE OTITIS MEDIA
}

\author{
Marwah Faisal Abd Ali* \& Isam M Al-Shareda ${ }^{@}$ \\ *MB,ChB, FIBMS Specialist in ORL-H\&N Surgery, Basrah Teaching Hospital. ${ }^{\circledR}$ MB,ChB, FIBMS, \\ Professor of ORL-H\&N Surgery, Department of Surgery, College of Medicine, University of Basrah, \\ Basrah, IRAQ.
}

\begin{abstract}
Chronic Otitis Media (COM) is a long standing inflammation of the middle ear and mastoid, it is a common health problem. The evidence of microbiology in COM in different studies showed variable frequencies, but little studies showed the role of fungal colonization.

This study aimed to identify the microbiology of cholesteatomatous COM concentrating on clarification of fungal colonization. A prospective study was done in otolaryngology department of Basrah Teaching Hospital from June 2018 till July 2019. This study was carried on a randomly collected data of 25 patients diagnosed as COM by a specific designed questionnaire including history and examination. The specimen was collected during mastoid surgery which included any cholesteatoma in the middle ear and mastoid and sent for bacteriological and mycological examination. Statistical study was done by using SPSS v.23

Bacteria were isolated in $84 \%$ of the cases in which Pseudomonas aeruginosa was the commonest one (33.3\%). Fungi were isolated in $28 \%$ cases, in which the Aspergillosis was the commonest $(19.3 \%)$. There is no recognizable pattern of significant association between the fungi and the reported complications or the stage of the disease.

In conclusion, there is significant percentage of fungal colonization in COM with cholesteatoma so it may has a role in inflammatory response of perimatrix, but there is no clear mechanism of this interaction, so may need larger sample and longer duration of study with postoperative follow-up and trail of combined antibiotic-antimycotic treatment.

Key words: clinical,microbiology, cholesteatoma, mastoid surgery, otitis media.
\end{abstract}

\section{Introduction}

- ntire forms of otitis media are Considered as a common health problem. Chronic suppurative otitis media (CSOM) is defined as a long standing inflammation in the middle ear and mastoid $^{1}$. The most frequent causative organisms in CSOM are; Pseudomonas aeruginosa, Methicillin sensitive Staph. aureus (MSSA), Methicillin resistant staphylococcus aureus (MRSA) and coagulase negative staphylococci with a variable frequency according to different studies ${ }^{2-5}$. Other bacteria cultured from chronic draining ears are: Enterobacter, Acinebacter, Proteus, Streptococcus, and Klebsiella. There is evidence of microbiology changing overtime, a study showed increasing in MRSA in CSOM from 0.7\% to $11.4 \%$ in a period between 1998$2006^{6}$. In chronic diseases such as chronic rhinosinusitis as a classical example in ENT, this shows susceptibility for fungal colonization $^{7}$. The usage of topical steroids as a combined topical treatment for CSOM is considered as an important predisposing factor for fungal colonization. Cholesteatoma itself is regarded as an ideal medium for saprophytic fungi because of its keratinous debris content ${ }^{8-12}$.

This study was designed to identify the microbiology of cholesteatomatous CSOM by using appropriate mycological and bacteriological methods in a group of operated upon patients, and concentrated 
on clarification of the clinical effects of the fungal colonization of cholesteatoma.

\section{Patients and methods}

This prospective study was done in the otolaryngology department at Basrah Teaching Hospital in the period from June 2018 to July 2019. The study sample composed of 25 patients of either gender, their ages ranged from 8-58 years old, the mean is 25 years. The data was collected according to a specific questionnaire. All patients were diagnosed as chronic otitis media by history of the most presenting symptoms and its duration, any complications, congenital anomalies such as cleft palate, chronic medical disease or immunocompromising disease, and preoperative treatment (before 1 week or less). Ear examination included; preoperative auroscope and otomicroscopy to classify the type of COM, fistula test, hearing assessment by tuning fork, PTA, and CT scan. Informed consent was mandatory. The type of surgery performed for patients was determined according to the disease extension and the opinion of the surgeon between mainly; Canal wall down (CWD) and canal wall up (CWU). For staging of cholesteatoma, EAONO/JOS system was used ${ }^{9}$ which applies to four types of cholesteatoma in the middle ear: Localized in the primary site, involving 2 or more sites, associated with extracranial complications, and associated with intracranial complications.

During mastoid surgery, a sterile micro forceps was used to collect the cholesteatoma and maintained in a sterile containers of normal saline, the sample was transported immediately to the mycology laboratory, Collage of Science, University of Basrah. Some samples were sent to the central laboratory, Basrah Teaching Hospital for processing. Fungal analysis was done by direct study of sample onto sterile glass slide containing a drop of $10 \% \mathrm{KOH}$, and culture onto sabouraud dextrose agar (SDA) containing $0.05 \mathrm{~g} / \mathrm{l}$ chloramphenicol. For analysis of bacteria, specimen was inoculated onto nutrient agar, blood agar, and MacConkey agar.

\section{Results}

A 25 patients diagnosed as CSOM with cholesteatoma were recruited in this study, $15(60 \%)$ were males and $10(40 \%)$ were females. All patients complained from otorrhoea, 11 of them (44\%) were associated with hearing defect, 3 cases have vertigo, and earache in one case (table I).

Table I: Presenting symptoms number and percentage

\begin{tabular}{|l|l|l|}
\hline Symptoms & No. & Percentage \\
\hline Otorrhoea & 10 & $40 \%$ \\
\hline Otorrhoea with hearing defect & 11 & $44 \%$ \\
\hline Otorrhoea with vertigo & 3 & $12 \%$ \\
\hline Otorrhoea with earache & 1 & $4 \%$ \\
\hline Total & 25 & $100 \%$ \\
\hline
\end{tabular}

Extracranial complications were demonstrated in $28 \%$ of the cases, mostly was labyrinthine fistula in $4(16 \%)$, facial palsy in $2(8 \%)$, and post auricular abscess in one.

Table II: Complications number and percentage

\begin{tabular}{|l|l|l|}
\hline Complications & No. & $\%$ \\
\hline No complication & 18 & $72 \%$ \\
\hline Facial palsy & 2 & $8 \%$ \\
\hline Labyrinthine fistula & 4 & $16 \%$ \\
\hline Post auricular abscess & 1 & $4 \%$ \\
\hline Total & 25 & $100 \%$ \\
\hline
\end{tabular}


According to EAONO/JOS system for cholesteatoma staging, we found 6 cases (24\%) of stage I, 12 cases (48\%) of stage II, and 7 cases (28\%) with stage III (Fig.1).

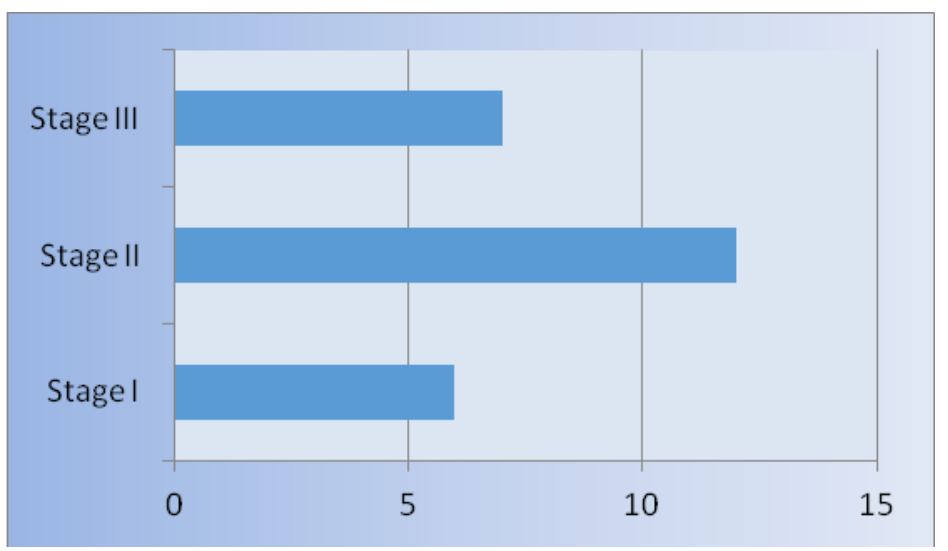

Figure 1: Number of disease stage according to EAONO/JOS system

Out of the suspicious 25 patients; 21 (84\%) confirmed to have bacterial cholesteatomatous CSOM with a positive culture. A total of 23 isolates representing eleven species of bacteria were obtained from the 21 patients. The most prevalent species was Pseudomonas aeruginosa (9 isolates, 39.13\% of total positive sample), followed by Enterobacter (4 isolates, 17.39\%), Eschericia coli (2 isolates, 8.69\%), and no bacterial growth in four patients ( $16 \%$ of the total sample) as shown in table III.

Table III: Types of the isolated bacteria

\begin{tabular}{|l|l|l|}
\hline Bacterial species & No. isolates & Percentage \\
\hline Acromobacter denitrificans & 1 & 4.34 \\
\hline Enterobacter sp & 4 & 17.39 \\
\hline Escherichia coli & 2 & 8.69 \\
\hline Klebsiella pneumonia & 1 & 4.34 \\
\hline Proteus sp & 1 & 4.34 \\
\hline Providenica struati & 1 & 4.34 \\
\hline Pseudomonas aeruginosa & 9 & 39.13 \\
\hline Ralstonia sp & 1 & 4.34 \\
\hline Staphylococcus aureus & 1 & 4.34 \\
\hline Staph. Lugdunensis & 1 & 4.34 \\
\hline Stenotrophomonas maltophile & 1 & 4.34 \\
\hline Total & 23 & 100 \\
\hline
\end{tabular}

There is no significant association between bacterial infection and the occurrence of complications ( P-value 0.999), neither with stage of disease (P-value 0.378) (table IV).

Table IV: Summary of association of bacterial infections with complications

\begin{tabular}{|l|l|l|l|l|}
\hline \multicolumn{2}{|c|}{} & \multicolumn{3}{|c|}{ Complications } \\
\cline { 2 - 5 } \multicolumn{2}{|c|}{} & Yes & No & Total \\
\hline Bacterial infection & Yes & 7 & 14 & 21 \\
\cline { 2 - 5 } & No & 0 & 4 & 4 \\
\cline { 2 - 5 } & Total & 7 & 18 & 25 \\
\hline
\end{tabular}

Fisher's exact test value: 0.242, P-value 0.999.

Regarding fungi, out of the 25 clinically diagnosed cholesteatomatous CSOM patients, only 7 cases were mycologically positive by direct microscopy and culture, which represent $28 \%$ of the total sample. In the current study, three genera were isolated; Aspergillus ( 3 species) that represent mold, candida (1 species), an ascomycetous yeast, 
and Naganishia (1 species), basidious yeast. The commonest species isolated in this study was Aspergillus niger ( 3 species, $37.5 \%$ of total positive sample) followed by Candida albicans $(2,25 \%)$. While A. flavus, A.fumigatus and Naganishia albida were represented by one isolate $(12.5 \%)$ for each species. Five cases were associated with bacterial isolation, and 2 cases as pure growth of fungi as demonstrated in table $\mathrm{V}$.

Table V: The isolated fungi number of isolates and their percentage

\begin{tabular}{|l|l|l|}
\hline Fungal species & No. isolates & Percentage \% \\
\hline Aspergillus flavus & 1 & 12.5 \\
\hline Aspergillus fumigatus & 1 & 12.5 \\
\hline Aspergillus niger & 3 & 37.5 \\
\hline Candida albicans & 2 & 25 \\
\hline Naganishia albida & 1 & 12.5 \\
\hline Total & 8 & 100 \\
\hline
\end{tabular}

Regarding association between fungi and the reported complications, there was no recognizable pattern of significant association (P-value 0.786)(table VI).

Table VI: Summary of association of fungal infections with complications

\begin{tabular}{|l|l|l|l|l|}
\hline \multicolumn{2}{|c|}{} & \multicolumn{3}{|c|}{ Complications } \\
\cline { 3 - 5 } \multicolumn{2}{|c|}{} & Yes & No & Total \\
\hline Fungal infection & Yes & 3 & 4 & 7 \\
\cline { 2 - 5 } & No & 4 & 14 & 18 \\
\cline { 2 - 5 } & Total & 7 & 18 & 25 \\
\hline
\end{tabular}

Chi squared value: $1.064, \mathrm{P}$-value 0.302

\section{Discussion}

In this study, the sample size was 25 patients of either gender with male preponderance, which is smaller than Singh $^{11}$ (40 patients) and more than Effat $^{13}$ (18 patients), with nearby same age groups in both studies.

In this study all patients complained from otorrhoea, in 11 patients associated with hearing loss, 3 patients with vertigo which are similar to Singh study. Because a comparison was done in 1999 between recovered micro-organism by conventional ear swab and by middle ear specimen, only $51 \%$ of middle ear aspirate micro-organisms were found in conventional ear swab, so we choose cholesteatoma as a middle ear or mastoid specimen for microbiology.

The proportion of different isolated micro-organisms varies from one study to another, but in general the Pseudomonas aeruginosa is the commonest isolated organism in about $39.13 \%$ cases in this study, $\left(31 \%\right.$ Ricciardiello et al ${ }^{10}, 28.6 \%$
Singh $^{11}$, and $51 \%$ Attallah $^{12}$. There was no bacterial growth in $16 \%$ of the specimen in this study which is corresponding to Attallah ${ }^{12}$. Regarding mycology, its incidence in cholesteatomatous CSOM is variable as low as $1.4 \%$ in Ricciardiello study ${ }^{10}$ to $89 \%$ in $\mathrm{Effat}^{13}$.

In this study, the fungal infestation was detected in 7 of our 25 cases (28\%), 8 fungal specious were isolated, Aspergillus is the commonest one $(62.5 \%)$ followed by candida in $25 \%$. Six of fungal isolates was associated with bacterial growth, while 2 isolated as pure nature.

The route of fungal infection to cholesteatoma $^{11}$ may be from external auditory canal or Eustachian tube. It can be induced by; topical steroid which is used in COM treatment, long term of antibiotics use, and chronic ear discharge which can cause meatal epithelium maceration which act as a good 
environment for colonization of fungi. It is difficult to distinguish whether fungal infestation in cholesteatoma has a clinical relation or just colonization. Capability of fungi to be opportunistic pathogens depends on the virulence factor which determined by: Toxin production, Size of the organism, Ability to grow in 37 centigrade at natural PH. Hall \& Farrior ${ }^{14}$ divided Aspergillus infestation in temporal bone into: localized, noninvasive, bony invasion by fibrosis and granulomatous respond, fulminant tissue and angioinvasion in immunocompromised persons.

The cholesteatoma is a sac of keratin surrounded by matrix and peri-matrix. The bacterial infection cause a chronic inflammation in a peri-matrix, so by the presence of pathogenic fungi can cause progression of $\mathrm{COM}$ by one of these mechanisms: mycotoxicosis of a toxic metabolites of fungi, fungi and bacteria interaction against antimicrobial and the host defense, pro-inflammatory cytokines which are released by common saprophytic fungi, hypersensitivity reaction type I \& III, and epithelial cell desquamation by fungal products. In this context, extracranial complications were recorded in 7 cases and fungal infection was detected in 3 cases with a $\mathrm{P}$-value 0.75 which is found to be not significant. This study fail to find a significant relationship between fungal infestation and complications and this result is similar to Singh study (P-value 0.68), however 18 patients of the sample were not having a complication, 14 (77.78\%) of them were not infected with fungi.

Conclusion; This study showed Gramnegative bacteria mainly Pseudomonas aeruginosa is significantly associated with COM. Also there was no insignificant percentage of fungus infection in cholesteatomatous COM which may play a significant role in an inflammatory response in the perimatrix and disease progression, but there is no clear mechanism of interaction between chronic inflammation and fungal infection.

\section{References}

1.Mittal A, Mann S, Panda NK, Mehra Y, Talwar P. Secondary fungal infections in chronic suppurative otitis media. Indian Journal of Otolaryngology \& Head and Neck Surgery. 1997;49(2):112-6.

2.Ahn JH, Kim M-N, An YS, Moon BJ. Preoperative, intraoperative, and postoperative results of bacterial culture from patients with chronic suppurative otitis media. Otology \& Neurotology. 2012;33(1):54-9.

3.Shim H, Park C, Kim M, Lee S, Yeo S. A pre-and postoperative bacteriological study of chronic suppurative otitis media. Infection. 2010;38(6):447-52.

4.Weckwerth PH, de Magalhaes Lopes CA, Duarte MAH, Weckwerth ACVB, Martins CHF, Neto DL, et al. Chronic suppurative otitis media in cleft palate: microorganism etiology and susceptibilities. The Cleft Palate-Craniofacial Journal. 2009;46(5):461-7.

5.Yeo SG, Park DC, Hong SM, Cha CI, Kim MG. Bacteriology of chronic suppurative otitis media-a multicenter study. Acta oto-laryngologica. 2007;127(10):1062-7.

6.Park MK, Jung MH, Kang HJ, Woo J-S, Lee H-M, Jung HH, et al. The changes of MRSA infections in chronic suppurative otitis media. Otolaryngology-Head and Neck Surgery. 2008;139(3):395-8.

7.Healy DY, Leid JG, Sanderson AR, Hunsaker DH. Biofilms with fungi in chronic rhinosinusitis. OtolaryngologyHead and Neck Surgery. 2008;138(5):641-7.

8.Bottone EJ, Hong T, Zhang DY. Basic mycology underscoring medically important fungi. Otolaryngologic clinics of North America. 1993;26(6):919-40.

9.Yung M, Tono T, Olszewska E, Yamamoto Y, Sudhoff H, Sakagami M, et al. EAONO/JOS joint consensus statements on the definitions, classification and staging of middle ear cholesteatoma. J Int Adv Otol. 2017;13(1):1-8. 10.Ricciardiello F, Cavaliere M, Mesolella M, Iengo M. Notes on the microbiology of cholesteatoma: clinical findings and treatment. Acta otorhinolaryngologica italica. 2009;29(4):197.

11.Singh GB, Solo M, Kaur R, Arora R, Kumar S. Mycology of chronic suppurative otitis media-cholesteatoma disease: An evaluative study. American journal of otolaryngology. 2018;39(2):157-61.

12.Attallah MS. Microbiology of chronic suppurative otitis media with cholesteatoma. Saudi medical journal. 2000;21(10):924-7.

13.Effat K, Madany N. Mycological study on cholesteatoma keratin obtained during primary mastoid surgery. The Journal of Laryngology \& Otology. 2014;128(10):881-4.

14.Hall PJ, Farrior JB. Aspergillus mastoiditis. Otolaryngology, Head and Neck Surgery. 1993;108(2):167-70. 